\title{
Adipose Tissue: A Tertiary Lymphoid Organ: Does It Change with Age?
}

\author{
Daniela Frasca $^{\mathrm{a}}$ Bonnie B. Blomberg ${ }^{\mathrm{a}}$ b \\ ${ }^{a}$ Department of Microbiology and Immunology, University of Miami Miller School of Medicine, Miami, FL, USA; \\ ${ }^{b}$ Sylvester Comprehensive Cancer Center, University of Miami Miller School of Medicine, Miami, FL, USA
}

\section{Keywords}

Aging $\cdot$ Adipose tissue $\cdot$ Inflammaging $\cdot$ Immunosenescence

\begin{abstract}
In this manuscript, we summarize published results showing that obesity and aging are inflammatory conditions associated with serious health problems, increased risk for disease and death. We show that fat mass increases with age and represents a major contributor to insulin resistance and the metabolic syndrome. We summarize the effects of age on the adipose tissue (AT), related to the abundance, distribution, cellular composition, endocrine signaling and function of the tissue. The AT is an immunological tissue, with several hallmarks of innate and adaptive immune responses. We show that in both mice and humans, the AT is heavily infiltrated by immune cells that have receptors for pro-inflammatory cytokines and chemokines secreted by the adipocytes and also by the immune cells that have infiltrated the AT. We also show that the AT provides an environment for the secretion of IgG antibodies with anti-self (autoimmune) reactivity. As we have previously shown, this is due to the release of self antigens following cell death due to hypoxia, as well as to the expression of activation-induced cytidine deaminase, the enzyme of class switch recombination, and the transcription factor T-bet by the resident $B$ cells, which also express the membrane marker CD11c, both involved in the production of autoimmune IgG antibodies. We show data in support of the AT as a tertiary lymphoid organ (TLO),
\end{abstract}

showing the examples of TLOs that develop within the AT, such as fat-associated lymphoid clusters and milky spots, as well as artery TLOs that develop in the adventitia areas of the aorta.

(c) 2019 S. Karger AG, Basel

\section{Inflammaging and Immunosenescence}

Aging is characterized by low-grade chronic inflammation called inflammaging [1], which represents a significant risk factor for morbidity and mortality of elderly individuals as it is implicated in the pathogenesis of several disabling diseases of old age. Inflammaging induces intrinsic inflammation in immune cells leading to decreased protective responses against infections and decreased vaccine responses. Levels of serum TNF- $\alpha$ have been shown to negatively correlate with $\mathrm{B}$ - and T-cell function. Results from our laboratory have shown that the age-related increase in plasma TNF- $\alpha$ induces TNF- $\alpha$ production by unstimulated B cells, without any antigenic stimulation and that this "pre-activated" phenotype of the $B$ cells renders them incapable of being optimally stimulated by exogenous antigens, mitogens or vaccines [2]. Moreover, B-cell function can be significantly increased by adding an anti-TNF- $\alpha$ antibody to cultured B lymphocytes [2], similar to what has also been shown for T cells [3]. The frequencies of CD4+CD28-T cells, which represent a pro-inflammatory CD4 T-cell subset with

\section{KARGER}

(c) 2019 S. Karger AG, Basel 
characteristics of immunosenescence, are increased in the blood of elderly individuals and these frequencies are also associated with high serum levels of TNF- $\alpha$ [4]. Mechanistically, it has been demonstrated that TNF- $\alpha$ inhibits the activity of the CD28 promoter in CD4+ T cells. Also, the frequencies of the pro-inflammatory CD8+CD28- T-cell subset have been shown to be increased in aging and associated with intrinsic cell inflammation [5].

Several factors contribute to inflammaging. These include polymorphisms in the promoter regions of pro-inflammatory genes, chronic stimulation of immune cells with viruses (cytomegalovirus), changes in gut microbiome composition and increased intestinal permeability (reviewed in [6]). Cellular senescence is another significant contributor to inflammaging due to the acquisition of the senescence-associated secretory phenotype by different cell types including immune cells. The senescenceassociated secretory phenotype is responsible for the secretion of pro-inflammatory chemokines, cytokines, growth factors and matrix metalloproteinases [7]. The age-dependent accumulation of senescent cells represents a favorable environment for the development of inflammatory-based age-related diseases.

Inflammaging may also be driven by the increase in adiposity with age [8], demonstrating the importance of adipose tissue (AT) inflammation in aging. The AT is the largest organ in humans and therefore age-related changes not only in AT composition and function, but also in AT metabolism, may lead to significant systemic changes, which in turn may accelerate and exacerbate the aging process. Changes in the lifestyle of elderly individuals as they start retirement, as well as changes in food choices and eating patterns, are responsible for the excessive accumulation of fat in different tissues. Obesity is considered to be an inflammatory condition associated with chronic activation of the innate immune system, responsible for pathologic conditions of old age such as Type-2 Diabetes, cancer, psoriasis, atherosclerosis and inflammatory bowel disease.

\section{Effects of Aging on the AT}

The AT undergoes significant changes during aging. Major changes are summarized in Table 1. These include abundance, distribution, cellular composition and endocrine signaling of the tissue. Fat mass increases with age in humans $[9,10]$ and mice $[9]$ and this is associated with increased low-grade chronic inflamma-
Table 1. Major changes in the human adipose tissue during aging

\begin{tabular}{ll} 
Type of change & Reference \\
\hline Increased total body fat & {$[8-10]$} \\
Increased visceral fat & {$[10]$} \\
Decreased subcutaneous fat & {$[10]$} \\
Increased ectopic fat distribution in different organs & \\
$\quad$ Liver & {$[12]$} \\
$\quad$ Skeletal muscle & {$[13]$} \\
$\quad$ Epicardium & {$[15]$} \\
$\quad$ Pancreas & {$[14]$} \\
$\quad$ Kidney & {$[11]$} \\
$\quad$ Blood vessels & {$[16]$} \\
Increased adipocyte size & {$[10,23]$} \\
Increased infiltration of macrophages & {$[10,23]$} \\
Increased expression of mRNA for pro-inflammatory & \\
$\quad$ cytokines & {$[18]$} \\
Increased accumulation of cells expressing the SASP & {$[9]$} \\
Increased secretion of leptin and decreased secretion & {$[10]$} \\
of adiponectin &
\end{tabular}

SASP, senescence-associated secretory phenotype.

tion, inflammaging [1], which contributes to metabolic dysfunction and development of insulin resistance (IR) that also increases with age. Moreover, an age-associated increase in the ectopic deposit of triglycerides in several tissues (liver, muscle, heart, pancreas, kidney) [1115] and in blood vessels [16] occurs, and this is associated with the development and/or progression of age-associated diseases.

Computational tomography scans have shown that with age, subcutaneous AT (SAT) mass decreases, whereas visceral AT (VAT) mass increases [10]. Studies conducted in mice have shown increased fat mass with age, with an increase in VAT, similar to elderly humans. In humans and mice, SAT and VAT are biologically distinct in terms of secretion of pro-inflammatory mediators, with VAT being more inflammatory. Furthermore, the secretion of adipokines from AT is regulated by nutrients, and these are increased with aging [17].

Aging significantly affects AT function by changing the profile of inflammatory mediators produced by the adipocytes, modifying pre-adipocyte number and function and increasing the infiltration of macrophages and other lymphocytes in the AT [10]. Studies in mice have indeed shown that the VAT of old mice, as compared to the VAT of young mice, is characterized by significantly higher expression of RNA for pro-inflammatory cytokines (IL-1 $\beta$, IL-6, TNF- $\alpha$ ) as well as COX-2, suggesting that obesity accelerates the aging process by inducing in- 
flammaging [18]. In these studies, obesity was shown to induce high levels of inflammation in macrophages from both the VAT and the peritoneum, but this happened only in young and not in old mice. Therefore, the hypothesis that obesity superimposed on aging would result in higher inflammation may not be correct, at least in mice, as young and old mice may respond differently to dietinduced obesity. Contrary to the above studies conducted in mice, our human results have shown that obesity decreases B-cell function and vaccine responses in both young and elderly individuals, and we found that obesity superimposed on aging drastically increases chronic lowgrade inflammation, leading to decreased specific immune responses [19]. Therefore, obesity represents an additional risk factor for the older population in which the prevalence of chronic disease as well as the occurrence of complications increases.

A variety of endogenous substances or stimuli, such as hypoxia, excess nutritional elements like Free Fatty Acids (FFAs) or products of cell death, have been shown to trigger chronic low-grade inflammation in the AT. The agerelated dysfunction of the AT, which includes increased inflammation, is associated with shorter lifespan and increase in the prevalence of age-related diseases, including cancer and dementia.

Lipolysis is the hydrolysis of triglycerides that occurs in the AT and generates FFAs and glycerol. In vitro studies have shown that the stimulation of lipolysis in the lean AT with catecholamines decreases with age, although catecholamine signaling in adipocytes is normal during aging. The mechanism proposed is that AT macrophages regulate age-related reduction in adipocyte lipolysis by lowering the bioavailability of norepinephrine due to the upregulation of genes controlling catecholamine degradation in an NLRP3 inflammasome-dependent manner. Deletion of NLRP3 in aging restored catecholamine-induced lipolysis through the downregulation of growth differentiation factor-3 and monoamine oxidase-a, which is known to degrade norepinephrine [20].

The AT is a site of accumulation of senescent cells [9], following oncogene activation, or metabolic stress. Not only senescent cells, but also other markers of aging increase in obesity. An example is telomeres, which are key markers of biological aging and are predictive biomarkers of effective immune responses. Shorter telomeres have been found in fat-derived cells and have been associated with increased BMI, waist:hip ratio and VAT accumulation, confirming that obesity may accelerate the aging process.

\section{The AT Is an Immunological Organ}

The AT is an immunological organ, composed of adipocytes and the Stromal Vascular Fraction, a mixture of mesenchymal, endothelial and immune cells. In both mice and humans, the lean AT has been shown to contain macrophages, T cells (T regulatory cells [Treg] and Th2), $\mathrm{B}$ cells, iNKT and eosinophils. Most of the AT macrophages are tissue-resident cells, established during early embryonic development and maintained at later ages independently of the infiltration of blood monocytes [21]. Studies in mice have shown that tissue-resident macrophages represent a heterogeneous population of cells characterized by unique membrane markers. These include pre vasculature-associated adipose macrophages (VAMs; CD11b+CD206 ${ }^{\mathrm{LOW}} \mathrm{MHCII}^{\mathrm{HI}} \mathrm{CD} 64+\mathrm{CD} 11 \mathrm{c}-$ ), VAM1 (type 1 macrophages, CD11b+CD206 ${ }^{\mathrm{HI}} \mathrm{MHCII}-$ ${ }^{\mathrm{HI}} \mathrm{Tim} 4^{\mathrm{INT}}$ ), VAM2 (type 2 macrophages, $\mathrm{CD} 11 \mathrm{~b}+\mathrm{CD}$ $206^{\mathrm{HI}} \mathrm{MHCII}^{\mathrm{INT}} \mathrm{Tim}^{\mathrm{HI}}$ ), double-positive macrophages (DP, CD11b + CD206 $6^{\mathrm{LOW}} \mathrm{MHCII}^{\mathrm{HI}} \mathrm{CD} 64+\mathrm{CD} 11 \mathrm{c}+$ ) [22]. Their names arise from their tight association with blood vessels. The different subpopulations of VAMs are capable of dynamically adapting to AT environment, metabolic stress and inflammatory stimuli, suggesting their role in tissue homeostasis. For example, high-fat diet or acute inflammatory stress induces the expansion of DP macrophages, almost absent in the lean AT. These macrophages are characterized by anti-inflammatory and repair gene signatures and have poor endocytic activity. Fasting rapidly and reversibly reduces not only their frequencies but also those of all subpopulations of VAMs.

Also, the other immune cell types in the AT are mostly localized around crown-like clusters and in perivascular spaces. In general, the cellular composition of the lean AT is skewed towards an anti-inflammatory phenotype with M2-like macrophages, Treg and Th2 being the predominant cell types, responsible for the secretion of antiinflammatory cytokines [23]. Group 2 innate lymphoid cells (ILC2s) are also present in lean AT where they contribute to AT remodeling, counteracting the inflammatory effect of obesity and inducing browning of white AT, which prevents diet-induced obesity. During the development of obesity, immune cell infiltration significantly increases. Exposure of macrophages to Danger- and Pathogen-Associated Molecular Patterns leads to the polarization of macrophages to an M1-like phenotype [24]. Also, the infiltration of Th1/Th17/T $\gamma \delta$ and of IFN- $\gamma$ producing CD8+ T cells, as well as B cells and ILC1s, significantly increases during obesity and contributes to the secretion of pro-inflammatory cytokines from M1 mac- 


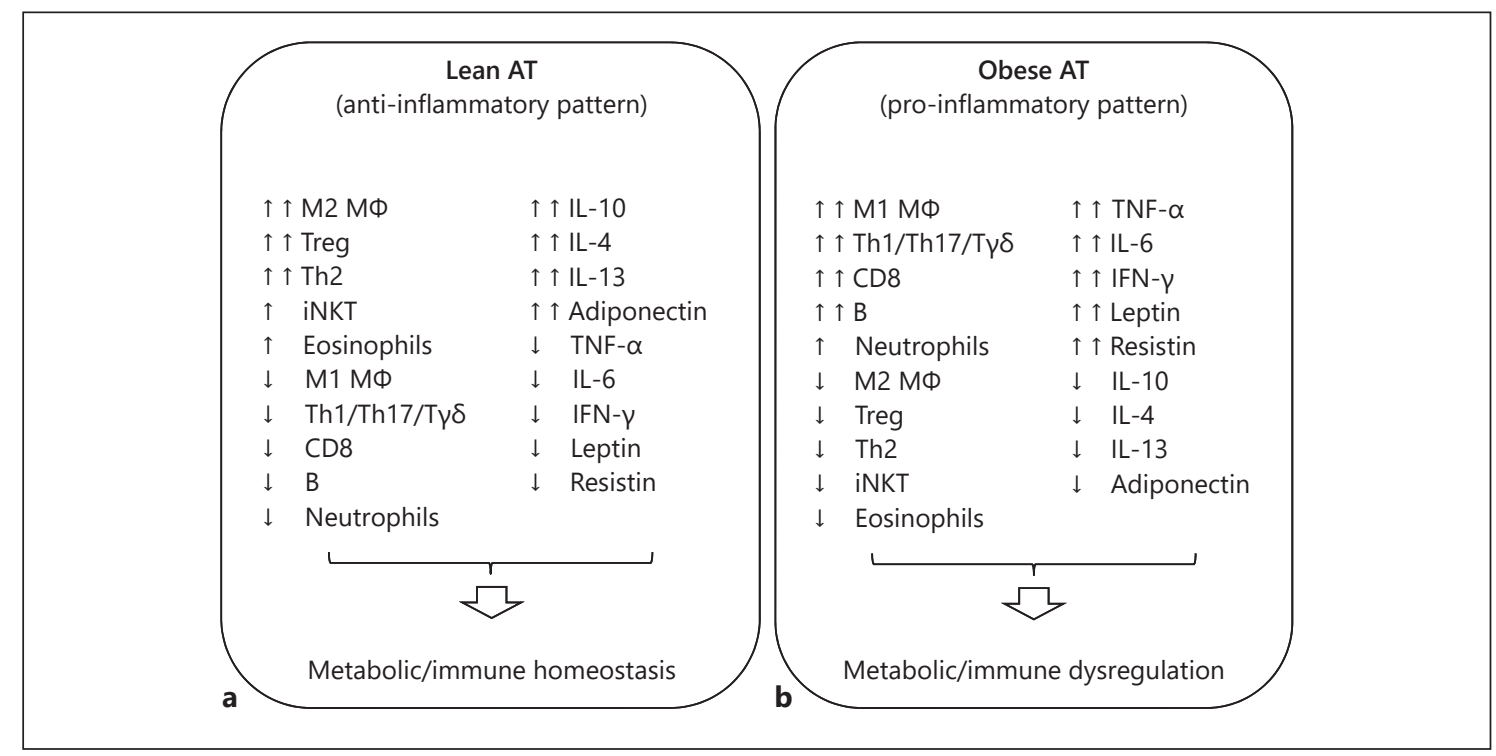

Fig. 1. Cellular and molecular composition of the lean and the obese ATs. The lean AT (a) is anti-inflammatory, characterized by small adipocytes and by a stromal vascular fraction containing M2-like macrophages (M $\Phi)$, Treg, Th2, B cells, iNKT, and eosinophils. These cells are responsible for the secretion of the anti-inflammatory cytokines IL-10, IL-4, IL-13, and the adipokine adiponectin. ILC2s are also present in lean AT. The obese AT (b), conversely, is characterized by larger adipocytes and has a

rophages and from infiltrating cells, local and systemic IR [23]. The cellular and molecular composition of the lean versus obese AT is shown in Figure 1.

The increase in AT mass is a characteristic of obesity. The AT is not only a metabolically active organ and a storage organ for excess nutrients due to over nutrition, but also a major endocrine organ that regulates body metabolism through its interactions with insulin target organs. The AT can be the largest tissue in individuals with obesity. Obesity-induced changes of the AT induce remodeling of the tissue, which involves the expansion of the size of the adipocytes (hypertrophy), accumulation of extracellular matrix components, increased formation of new blood vessels, increased perfusion of capillaries and increased secretion of pro-inflammatory mediators (cytokines and chemokines), involved in the recruitment of immune cells to the AT. It has been proposed that the interaction of extracellular matrix molecules with receptors primarily expressed by adipocytes and macrophages contributes to AT inflammation, cell death and metabolic complications of obesity. Failure to undergo appropriate remodeling in response to over-nutrition is detrimental to body metabolic homeostasis, as excess nutrients pro-inflammatory phenotype with high frequencies of M1-like macrophages. The obese AT is heavily infiltrated with Th1/Th17/ $\mathrm{T} \gamma \delta$, CD8+ T cells, B cells, ILC1s, neutrophils. The secretion of pro-inflammatory cytokines by both the adipocytes (TNF- $\alpha$, IL-6) and the immune cells (TNF- $\alpha$, IL-6, IL-17, IFN- $\gamma$ ) and the secretion of the adipokines leptin and resistin by the adipocytes significantly contributes to metabolic and immune dysregulation leading to inflammaging and IR.

promote metaflammation, a process in which excess nutrients promote chronic low-grade inflammation, whose metabolic hallmarks are high levels of glucose, lipids, FFAs and reactive oxygen species.

Published data have shown that the obese AT of both mice and humans is heavily infiltrated by immune cells. Monocyte infiltration and macrophage expansion within the AT have been considered major drivers of inflammation due to the secretion of pro-inflammatory cytokines and chemokines involved in the recruitment of immune cells. Other immune cell types, however, also infiltrate the obese AT and contribute to the establishment of inflammatory conditions leading to IR. The immune cells are recruited to the AT by chemokines released by both adipocytes and infiltrating immune cells. Moreover, during the development of obesity, the supply of oxygen to the expanding AT becomes inadequate, generating areas of hypoxia, responsible for the activation of the transcription factor hypoxia-inducible factor- $1 \alpha$ and further release of pro-inflammatory cytokines. Hypoxia also induces cell death and release of "self" antigens, which stimulate class switch and the production of pathogenic IgG antibodies. In addition to hypoxia, DNA damage and NK 
cell cytotoxicity are additional mechanisms contributing to the release of "self" antigens in the obese AT, as we have recently shown [25].

TNF- $\alpha$, secreted in large amounts by both adipocytes and immune cells in the obese AT, stimulates lipolysis, the process of hydrolysis of tryglycerides that generate FFAs, glycerol and lipids, all capable of inducing local and systemic inflammation through NF-kB activation and activation of pro-inflammatory macrophages. FFAs also directly inhibit B-cell function (cytokine production) in vitro. Excessive lipolysis in the obese AT leads to increased oxidative stress that promotes DNA damage and AT inflammation.

Leptin is an adipokine primarily secreted by the AT [26]. Leptin, similar to other pro-inflammatory mediators secreted by the AT, is released into the circulation. Leptin has been shown to induce systemic inflammation and is known to downregulate functional immune responses. Leptin induces the secretion of inflammatory cytokines by macrophages, $\mathrm{T}$ cells and $\mathrm{B}$ cells in vitro, and leukotriene synthesis by alveolar macrophages, leading to pulmonary inflammation. Increased leptin is at least one of the mechanisms responsible for the reduced production of protective antibodies in response to vaccination [19] in individuals with obesity.

The AT also contributes to the increased production of autoimmune antibodies in mice [27] and in individuals with obesity [28]. It has been shown that obesity creates an optimal environment for autoimmune diseases, as it leads to a breakdown of mechanisms of anti-self-tolerance, and this happens in the context of a pro-inflammatory environment, which worsens disease progression. Mice fed a high-fat diet were shown to have higher plasma levels of heat shock protein 60 (HSP60), as compared to mice fed normal chow and these were associated with higher plasma levels of HSP60-specific IgG2c (autoimmune) antibodies. Studies conducted in humans have shown that the serum of IR obese individuals contains autoantibodies specific for intracellular proteins, ubiquitously expressed in immune cells as well in tissues including, pancreas, liver, muscle or AT, suggesting the release of "self" antigens in insulin target tissues. These IgG autoantibodies are present in the serum of IR individuals at a significantly higher extent as compared to insulin sensitive individuals. This result was obtained using a protein array in which $>9,000$ selected "self" antigens were probed with serum from IR and insulin sensitive individuals, overweight to obese, age- and BMI- matched. The majority of "self" antigens are intracellular proteins (Golgi and endoplasmic reticulum proteins, RNA polymerase, gluta- thione transferase, signaling proteins) with variable tissue expression.

We have recently shown that the human SAT is responsible for the secretion of fat-specific IgG antibodies and that SAT B cells express mRNA for activation-induced cytidine deaminase, the enzyme of class switch recombination and somatic hypermutation, as well as the transcription factor $\mathrm{T}$-bet and the membrane marker $\mathrm{CD} 11 \mathrm{c}$, both involved in the production of autoimmune IgG antibodies [25]. More recently, we have identified several "self" antigens that stimulate the secretion of these autoimmune antibodies in cultures of SAT-derived immune cells, using a Protein G immunoprecipitation protocol to purify the autoantibodies, combined with mass spectrometry. Results show that $25 \%$ of these "self" antigens are found at high frequency in all the individuals evaluated so far in independent experiments. Moreover, almost all (>90\%) of these "self" antigens (lipid and protein antigens, DNA, RNA) are cytoplasmic, nuclear or membrane associated [Frasca et al., manuscript in preparation].

\section{Tertiary Lymphoid Organs}

Lymphoid organs are classified as primary, secondary and tertiary lymphoid organs (TLOs). This hierarchy is not clearly defined because their function is very dynamic. Primary lymphoid organs are responsible for the generation of naïve B and T lymphocytes. Secondary lymphoid organs, SLOs, (lymph nodes, spleen, tonsils, mucosa-associated lymphoid tissues), are the sites of antigen-driven expansion of lymphocytes, generation of memory lymphocytes and plasma cells. Examples of inducible lymphoid tissues present at mucosal surfaces are bronchial-associated lymphoid tissues in the lung, gutassociated lymphoid tissues in the intestine, and nasopharyngeal-associated lymphoid tissues. Tertiary lymphoid organs, TLOs, also known as tertiary lymphoid tissues or ectopic lymphoid follicles, originate in adult peripheral tissues following continuous extravasation of leukocytes in the presence of persistent antigen, occurring at sites of infection, allograft rejection, autoimmune attack [29-32]. TLOs are highly organized structures and share many features with SLOs, including compartmentalization of $\mathrm{T}$ and $\mathrm{B}$ lymphocytes into $\mathrm{T}$-cell zones and $\mathrm{B}$-cell follicles, the presence of antigen-presenting cells, mainly follicular dendritic cells (FDCs), together with the presence of chemokines responsible for the recruitment and compartmentalization of the immune cells. TLOs are 
also characterized by the presence of extensive networks of fibroblast reticular cells in the T-cell zone, germinal centers (GCs) in the B-cell follicle, and markers of CSR such as activation-induced cytidine deaminase. However, even when all these criteria for definition of TLOs are not found, TLOs can still be identified [30].

Several experimental findings have characterized the mechanisms responsible for TLO formation, as reviewed in [29]. TLOs are sites for the generation of protective antibody responses at sites of infection. This is because, similar to SLOs, TLOs are enriched in B and T cells that accumulate on the network of FDCs, leading to the activation of $\mathrm{B}$ and $\mathrm{T}$ cells, GC formation, memory $\mathrm{B}$ cell generation and secretion of specific antibodies by plasma cells found in proximity of TLOs, as has been shown after viral or bacterial infections.

TLOs are also sites for the generation of self-reactive B and $\mathrm{T}$ cells, as suggested by their presence in models of chronic autoimmunity, transplant rejection, and their association with tissue destruction. Chronic inflammation induced by chronic infections, autoimmunity or allograft rejection has been shown to be often accompanied by TLO formation. TLOs in the kidneys of patients with Systemic Lupus Erythematosus, SLE, are enriched in GCs and show intrarenal B-cell clonal expansion and ongoing SHM, suggesting TLO neogenesis in the pathogenesis of SLE tubulointerstitial inflammation [33]. Moreover, GC numbers were positively associated with the presence of immune complexes, suggesting that tissue inflammation and damage is likely due to the interaction of renal antigens with locally generated autoantibodies able to fix complement [33]. Similar to SLE patients, TLOs in the synovium of Rheumatoid Arthritis patients are characterized by the accumulation of B cells able to present antigens to $\mathrm{T}$ cells, to secrete pro-inflammatory cytokines and induce pro-inflammatory T cells [34]. Pro-inflammatory Th17 cells have also been shown to accumulate in the synovium of rheumatoid arthritis patients and it has been proposed that TLOs could potentially help to support Th17 cell maintenance. In conclusion, although most of the published data support a pathogenic role for TLOs in autoimmune patients, TLOs may also have a protective effect, as in their absence pathogenic lymphocytes may enter the circulation and reach target tissues inducing deleterious tissue damage.

TLOs may have pathogenic or protective effects also in transplant patients. TLOs may contribute to chronic transplant rejection by generating both humoral and cellular local alloantigen-specific immune responses, but they have also been observed in long-term transplanted patients, suggesting that they might be able to regulate these responses. It has been shown that the majority of chronically rejected human kidney grafts show a highly organized microarchitecture with the infiltrating immune cells grouped in areas in which B cells and FDCs are surrounded by $\mathrm{T}$ cells and mature DCs, with adjacent plasma cells. TLOs have been observed in several chronically rejected organs, such as pancreas, livers, hearts and lungs [35].

Atherosclerosis, a chronic inflammatory disease of arterial walls, is associated with TLO formation. TLOs have been shown to have a pathogenic role on atherosclerosis with artery TLOs (ATLOs) participating in atherosclerotic-specific primary immune responses leading to autoantibody secretion [36]. ATLOs are aberrant lymphoid aggregates that harbor all immune cells needed for primary immune responses; they are organized into distinct compartments, containing T- and B-cell follicles, monocytederived DCs, lymphoid DCs and plasmacytoid DCs. The B-cell follicles contain proliferating centroblasts, centrocytes, mantle zone B cells and peripheral niches of plasma cells (mainly long-lived), as well as FDCs, all in organized GCs. Survival factors for long-lived plasma cells are highly expressed in ATLOs. ATLOs increase with age $[36,37]$. Studies conducted in mice deficient for low-density lipoprotein receptor (LDLR-/-) or for apolipoprotein E (ApoE-/-) mice have helped to characterize the structure of ATLOs, and it has been shown that old ApoE-/- mice develop ATLOs in adventitia areas of the aorta adjacent to atherosclerotic plaques, suggesting the generation of autoimmune responses within the diseased arterial wall during aging. Lymphotoxin (LT) $\beta$ receptor signaling has been shown to promote ATLOs organogenesis, and treatment of apoE(-/-) mice with LT $\beta$ R-Ig blocks LT $\beta$ R signaling, disrupting the structure and maintenance of ATLOs.

ATLOs have been found in the adventitia of patients with atherosclerotic aortic aneurysms and also with ischemic heart disease [38].

\section{Tertiary Lymphoid Organs Developing within the AT}

The age-associated increase in fat leads to the development of TLOs in various organ systems. TLOs have indeed not only been associated with inflammatory diseases but also seem to be generated during chronic inflammation. The AT is an inflammatory organ, characterized by chronic secretion of pro-inflammatory mediators such as cytokines that modulate adipocyte lipolysis and differ- 
entiation, thus supporting local inflammation. The AT also secretes several pro-inflammatory chemokines with potent chemo-attractant capacities for circulating immune cells. Several evidences suggest that the AT may be considered a TLO, including the high level of local inflammation, the high concentration of pro-inflammatory chemokines, the heavy infiltration of immune cells, the persistence of antigens that can stimulate the production of tissue-directed antibodies with a pathogenic role, and the presence of GCs. However, the AT, as opposed to TLOs, does not originate from continuous extravasation of leukocytes. Our results recently published showing that secretion of autoantibodies specific for fat antigens occur in the human SAT [25] may further support the AT as a TLO. TLO formation has indeed been shown to lead to the secretion of tissue-directed autoantibodies.

Fat-associated lymphoid clusters (FALCs) represent an example of TLOs that develop within VAT [39]. It has been hypothesized, but not experimentally proved yet, that increase in VAT with age leads to increased FALCs. FALCs are associated with adipocytes in mucosal surfaces (gonadal, mesenteric, omental, mediastinal and pericardial AT), but the frequency of FALCs varies among the different anatomical locations. FALCs are less organized structures as compared to TLOs and no discernible compartmentalization of $B$ and $T$ cells is observed. Immune cells in the FALCs include B1 and B2 B cells, T cells and different subsets of innate immune cells, especially ILC2s. FALCs play a role in regulating local immune responses. For example, FALCs have been shown to be involved in the retention of $\mathrm{B} 1 \mathrm{~B}$ cells in the peritoneal cavity, due to their high levels of $\alpha 4, \alpha 6$ and $\beta 1$ integrins, which allow their adherence to parietal and visceral endothelium of the cavity. When surface expression of these integrins is downregulated, B1 B cells egress from the peritoneal cavity, recruited by CXCL13-producing clusters in omentum, mesenteric lymph node and spleen [39]. FALC formation requires local inflammation and signaling through the TNF- $\alpha$ receptor. FALCs are essential for the function of innate B cells in cavities, mainly the production of polyreactive IgM antibodies for protection of the cavities, through the secretion of IL-5 and IL-33. FALCs are also present in the para-aortic AT that is adjacent to the adventitia suggesting that they may participate in atherosclerotic immune responses.

Data on milky spots is inconsistent/controversial. Milky spots are aggregates of leukocytes that contribute to peritoneal immunity by collecting antigens and pathogens from the peritoneal cavity. Depending on the stimulus, milky spots promote different immune responses, such as inflammation, tolerance and fibrosis. Milky spots, similar to TLOs, and FALCs in particular, are embedded in the omental tissue, highly enriched in B1 B cells, with fewer T cells and FDCs [40]. In general, B cells form a central cluster, surrounded by macrophages and DCs. The B cell attacking chemokine CXCL13, crucial for the recruitment and retention of $\mathrm{B}$ cells in the clusters, is abundant in milky spots and associated with DCs. T cells and ILCs can be together with the B cells or they may cluster around blood vessels. Milky spots support B- and Tcell immune responses to peritoneal antigens and they work similar to lymph nodes, as they collect and respond to local antigens. However, their cellular composition is more similar to that of the peritoneal cavity as compared to that of lymph nodes or other SLOs. Although a close examination has shown indiscernible differences between milky spots and FALCs in the mesenteric, mediastinal and gonadal AT, the absence of FDCs from milky spots, has suggested that they are not true TLOs. TLOassociated DCs are indeed the major source of chemokines and cytokines that support the long-term maintenance of TLOs and support adaptive immune responses through local T-cell priming and control of GC reactions. There are no reports on the effects of aging on the formation and function of milky spots.

\section{Conclusions}

In the AT many aging-associated processes occur, such as inflammaging, mitochondrial dysfunction and accumulation of senescent cells and therefore therapeutic interventions to treat obesity should also have a beneficial effect on aging and aging-associated conditions. Dietary interventions (caloric restriction) have shown direct beneficial effects on the AT, reducing inflammation, DNA damage, cellular senescence and ectopic lipid deposition, and increasing autophagy and DNA repair mechanisms. Caloric restriction has also been effective in preventing age-related changes in gene expression, thus leading to extended lifespan. Although still preliminary, these studies have indicated that the AT may be a valid target of therapies that alleviate age-associated conditions and improve health span.

\section{Acknowledgements}

This study was supported by NIH R56 AG32576 (D.F. and B.B.B.), R56 AG059719 (D.F.) and R01 AG023717 (B.B.B.). 


\section{References}

1 Franceschi C, Bonafè M, Valensin S, Olivieri F, De Luca M, Ottaviani E, et al. Inflamm-aging. An evolutionary perspective on immunosenescence. Ann N Y Acad Sci. 2000 Jun; 908(1):244-54.

2 Frasca D, Diaz A, Romero M, Landin AM, Blomberg BB. High TNF-a levels in resting B cells negatively correlate with their response. Exp Gerontol. 2014 Jun;54:116-22.

3 Parish ST, Wu JE, Effros RB. Modulation of T lymphocyte replicative senescence via TNF\{alpha\} inhibition: role of caspase-3. J Immunol. 2009 Apr;182(7):4237-43.

4 Bryl E, Vallejo AN, Weyand CM, Goronzy JJ. Down-regulation of CD28 expression by TNF-alpha. J Immunol. 2001 Sep;167(6): 3231-8.

5 Saurwein-Teissl M, Lung TL, Marx F, Gschösser C, Asch E, Blasko I, et al. Lack of antibody production following immunization in old age: association with CD8(+) CD28(-) T cell clonal expansions and an imbalance in the production of Th1 and Th2 cytokines. J Immunol. 2002 Jun;168(11):58939.

6 Frasca D, Blomberg BB. Inflammaging decreases adaptive and innate immune responses in mice and humans. Biogerontology. 2016 Feb;17(1):7-19.

7 Campisi J. Cellular senescence: putting the paradoxes in perspective. Curr Opin Genet Dev. 2011 Feb;21(1):107-12.

8 Forsythe LK, Wallace JM, Livingstone MB. Obesity and inflammation: the effects of weight loss. Nutr Res Rev. 2008 Dec;21(2): 117-33.

9 Tchkonia T, Morbeck DE, Von Zglinicki T, Van Deursen J, Lustgarten J, Scrable H, et al. Fat tissue, aging, and cellular senescence. Aging Cell. 2010 Oct;9(5):667-84.

10 Zamboni M, Rossi AP, Fantin F, Zamboni G, Chirumbolo S, Zoico E, et al. Adipose tissue, diet and aging. Mech Ageing Dev. 2014 MarApr;136-137:129-37.

11 Foster MC, Hwang SJ, Porter SA, Massaro JM, Hoffmann U, Fox CS. Fatty kidney, hypertension, and chronic kidney disease: the Framingham Heart Study. Hypertension. 2011 Nov;58(5):784-90.

12 Machann J, Thamer C, Schnoedt B, Stefan N, Stumvoll M, Haring HU, et al. Age and gender related effects on adipose tissue compartments of subjects with increased risk for type 2 diabetes: a whole body MRI/MRS study. MAGMA. 2005 Jul;18(3):128-37.

13 Ryan AS, Nicklas BJ. Age-related changes in fat deposition in mid-thigh muscle in women: relationships with metabolic cardiovascular disease risk factors. Int J Obes Relat Metab Disord. 1999 Feb;23(2):126-32.
14 Saisho Y, Butler AE, Meier JJ, Monchamp T, Allen-Auerbach M, Rizza RA, et al. Pancreas volumes in humans from birth to age one hundred taking into account sex, obesity, and presence of type-2 diabetes. Clin Anat. 2007 Nov;20(8):933-42.

15 Silaghi A, Piercecchi-Marti MD, Grino M, Leonetti G, Alessi MC, Clement K, et al. Epicardial adipose tissue extent: relationship with age, body fat distribution, and coronaropathy. Obesity (Silver Spring). 2008 Nov;16(11): 2424-30.

16 Robert L. Aging of the vascular-wall and atherosclerosis. Exp Gerontol. 1999 Jul;34(4): 491-501.

17 Einstein FH, Fishman S, Bauman J, Thompson RF, Huffman DM, Atzmon G, et al. Enhanced activation of a "nutrient-sensing" pathway with age contributes to insulin resistance. FASEB J. 2008 Oct;22(10):3450-7.

18 Wu D, Ren Z, Pae M, Guo W, Cui X, Merrill $\mathrm{AH}$, et al. Aging up-regulates expression of inflammatory mediators in mouse adipose tissue. J Immunol. 2007 Oct;179(7):4829-39.

19 Frasca D, Ferracci F, Diaz A, Romero M, Lechner S, Blomberg BB. Obesity decreases B cell responses in young and elderly individuals. Obesity (Silver Spring). 2016 Mar;24(3): 615-25.

20 Camell CD, Sander J, Spadaro O, Lee A, Nguyen KY, Wing A, et al. Inflammasomedriven catecholamine catabolism in macrophages blunts lipolysis during ageing. Nature. 2017 Oct;550(7674):119-23.

21 Davies LC, Jenkins SJ, Allen JE, Taylor PR. Tissue-resident macrophages. Nat Immunol. 2013 Oct;14(10):986-95.

22 Silva HM, Báfica A, Rodrigues-Luiz GF, Chi J, Santos PD, Reis BS, et al. Vasculature-associated fat macrophages readily adapt to inflammatory and metabolic challenges. J Exp Med. 2019 Apr;216(4):786-806.

23 Lumeng CN, Bodzin JL, Saltiel AR. Obesity induces a phenotypic switch in adipose tissue macrophage polarization. J Clin Invest. 2007 Jan;117(1):175-84.

24 Grant RW, Dixit VD. Adipose tissue as an immunological organ. Obesity (Silver Spring). 2015 Mar;23(3):512-8.

25 Frasca D, Diaz A, Romero M, Thaller S, Blomberg BB. Secretion of autoimmune antibodies in the human subcutaneous adipose tissue. PLoS One. 2018 May;13(5):e0197472.

26 Friedman JM, Halaas JL. Leptin and the regulation of body weight in mammals. Nature. 1998 Oct;395(6704):763-70.

27 Şelli ME, Wick G, Wraith DC, Newby AC. Autoimmunity to HSP60 during diet induced obesity in mice. Int J Obes. 2017 Feb;41(2): 348-51.
28 Versini M, Jeandel PY, Rosenthal E, Shoenfeld Y. Obesity in autoimmune diseases: not a passive bystander. Autoimmun Rev. 2014 Sep;13(9):981-1000.

29 Ruddle NH. High Endothelial Venules and Lymphatic Vessels in Tertiary Lymphoid Organs: Characteristics, Functions, and Regulation. Front Immunol. 2016 Nov; 7:491.

30 Neyt K, Perros F, GeurtsvanKessel CH, Hammad H, Lambrecht BN. Tertiary lymphoid organs in infection and autoimmunity. Trends Immunol. 2012 Jun;33(6):297-305.

31 Ruddle NH. Lymphatic vessels and tertiary lymphoid organs. J Clin Invest. 2014 Mar; 124(3):953-9.

32 Shipman WD, Dasoveanu DC, Lu TT. Tertiary lymphoid organs in systemic autoimmune diseases: pathogenic or protective? F1000 Res. 2017 Feb;6:196.

33 Chang A, Henderson SG, Brandt D, Liu N, Guttikonda R, Hsieh C, et al. In situ B cellmediated immune responses and tubulointerstitial inflammation in human lupus nephritis. J Immunol. 2011 Feb;186(3):184960.

34 Weyand CM, Goronzy JJ, Takemura S, Kurtin PJ. Cell-cell interactions in synovitis. Interactions between $\mathrm{T}$ cells and B cells in rheumatoid arthritis. Arthritis Res. 2000;2(6): 457-63.

35 Koenig A, Thaunat O. Lymphoid Neogenesis and Tertiary Lymphoid Organs in Transplanted Organs. Front Immunol. 2016 Dec; 7: 646.

36 Yin C, Mohanta SK, Srikakulapu P, Weber C, Habenicht AJ. Artery Tertiary Lymphoid Organs: Powerhouses of Atherosclerosis Immunity. Front Immunol. 2016 Oct; 7:387.

37 Srikakulapu P, Hu D, Yin C, Mohanta SK, Bontha SV, Peng L, et al. Artery Tertiary Lymphoid Organs Control Multilayered Territorialized Atherosclerosis B-Cell Responses in Aged ApoE-/- Mice. Arterioscler Thromb Vasc Biol. 2016 Jun;36(6):1174-85.

38 Akhavanpoor M, Gleissner CA, Akhavanpoor H, Lasitschka F, Doesch AO, Katus HA, et al. Adventitial tertiary lymphoid organ classification in human atherosclerosis. Cardiovasc Pathol. 2018 Jan - Feb;32:8-14.

39 Bénézech C, Luu NT, Walker JA, Kruglov AA, Loo Y, Nakamura K, et al. Inflammation-induced formation of fat-associated lymphoid clusters. Nat Immunol. 2015 Aug;16(8):81928.

40 Krist LF, Eestermans IL, Steenbergen JJ, Hoefsmit EC, Cuesta MA, Meyer S, et al. Cellular composition of milky spots in the human greater omentum: an immunochemical and ultrastructural study. Anat Rec. 1995 Feb; 241(2):163-74. 